Carolina Marques Olibon ${ }^{1}$

Marco Aurelo Knippel Galletta ${ }^{2}$

Rossana Pulcinel Vielra Francisco ${ }^{2}$

Marle dos Santos Alvarenga ${ }^{3}$

\section{Artigo Original}

Palavras-chave

Gravidez

Ganho de peso

Tradução

Estudos de validação

Keywords

Pregnancy

Weight gain

Translating

Validation studies

\title{
Adaptação transcultural da Escala de Atitudes em Relação ao Ganho de Peso na Gestação
}

\author{
Cross-cultural adaptation of the Pregnancy and \\ Weight Gain Attitude Scale
}

Resumo

OBJETIVO: Apresentar a adaptação transcultural para o português da Escala de Atitudes em Relação ao Ganho de Peso na Gestação. MÉTODOS: Essa escala, que contém afirmações que expressavam diferentes atitudes de gestantes em relação ao seu próprio ganho de peso, foi desenvolvida para determinar se as atitudes em relação ao corpo afetariam o ganho de peso durante a gestação. Os procedimentos foram: tradução, retrotradução, avaliação da compreensão, elaboração de versão final, aplicação da escala em 180 gestantes (média 29,6 anos e idade gestacional 25,7 semanas) e análise psicométrica. RESULTADOS: Constatou-se equivalência satisfatória entre as versões inglês-português e boa consistência interna (Alpha de Cronbach 0,7). A análise fatorial exploratória sugeriu quatro subescalas com variância total explicada de 51,4\%. CONCLUSÃO: A escala se demonstrou válida e pode ser utilizada em estudos com gestantes no Brasil para avaliação de atitudes em relação ao ganho de peso e detecção e prevenção de comportamentos disfuncionais durante a gestação.

Abstract

PURPOSE: To present the cross-cultural adaptation to Brazilian Portuguese language of the Pregnancy and Weight Gain Attitude Scale. METHODS: This scale was developed in order to verify whether attitude toward thinness affects weight gain during pregnancy and contains statements that express different attitudes of pregnant women regarding their own weight gain. The procedures were: translation, back translation, comprehension evaluation, preparation of a final version, application of the scale to 180 pregnant women (mean age=29.6, gestational age=25.7 weeks) and psychometric analysis. RESULTS: Satisfactory equivalence between the versions and satisfactory internal consistency (Cronbach's alpha 0.7) were detected. The exploratory factor analysis suggested four subscales with $51.4 \%$ total variance explained. CONCLUSION: The scale proved to be valid and can be used in studies with pregnant women in Brazil to assess attitudes toward weight gain and to detect and prevent dysfunctional behaviors during pregnancy.

Correspondêncio

Carolina Marques Olibon Avenida Doutor Arnaldo, $715-2^{\circ}$ andar CEP: $01246-904$

São Paulo (SP), Brasil

Recebido

$31 / 03 / 2014$

Aceito com modificaçōes

$03 / 07 / 2014$
Clínica Obstétrica do Hospital das Clínicas da Faculdade de Medicina da Universidade de São Paulo; e Faculdade de Saúde Pública da Universidade de São Paulo - USP - São Paulo (SP), Brasil.

IPrograma de Pós-graduação em Nutrição em Saúde Pública, Faculdade de Saúde Pública, Universidade de São Paulo - USP São Paulo (SP), Brasil.

2Departamento de Obstetrícia e Ginecologia, Faculdade de Medicina, Universidade de São Paulo - USP - São Paulo (SP), Brasil.

${ }^{3}$ Departamento de Nutrição, Faculdade de Saúde Pública, Universidade de São Paulo - USP - São Paulo (SP), Brasil.

Conflito de interesses: nada a declarar. 


\section{Introdução}

A gestação é caracterizada por transformações corporais relacionadas aos ritmos metabólicos e hormonais, demandando o processo de integração de uma nova imagem corporal que ocorre em um curto espaço de tempo. Essas alterações têm repercussões nas dimensões física e emocional, o que faz com que a gestação seja considerada um período de crise que exige da gestante uma resposta adaptativa ${ }^{1}$.

Embora seja um momento especial na vida da mulher, no contexto atual, com a agregação de novos papeis à vida feminina, sentimentos comuns como ansiedade $\mathrm{e}$ angústia podem ser potencializados pelas pressões sociais, trazendo sobrecarga de demandas e impedindo a mulher de desfrutar da realização da gravidez ${ }^{2}$. As mudanças corporais, apesar de naturais, como o ganho de peso, podem acarretar insatisfações, pois muitas mulheres apresentam dificuldade em aceitá-las, tornando-se inseguras quanto à permanência das alterações físicas e mais propensas à preocupação com a imagem corporal, em decorrência de fatores como pressão social para ser magra, comparação de seu corpo com o de outras mulheres, entre outros ${ }^{3}$.

Pesquisas que focaram os aspectos perceptivos da imagem corporal encontraram um tamanho corporal superestimado em mulheres americanas grávidas em comparação a mulheres não grávidas. As gestantes também se encontram mais expostas às cobranças sociais, principalmente da mídia .

A percepção da gestante em relação a seu abdômen é influenciada pelos corpos de modelos expostos pela mídia durante a gestação ${ }^{5}$. Trabalho mais recente investigou a representação das mudanças de peso e forma corporais durante a gestação e o período pós-parto de celebridades em websites de revistas de entretenimento americanas e encontrou que o peso ganhado pelas celebridades na gestação era mencionado mais frequentemente do que o peso gestacional em si. Além disso, mudanças no peso (ganho ou perda) receberam maior atenção do que o peso absoluto ${ }^{6}$. Esse estudo sugere uma lacuna preocupante entre o retrato da gestação e as experiências pós-parto das celebridades e de mulheres não famosas, que pode levar a expectativas irreais tanto para grávidas quanto para o público em geral.

Alguns estudos internacionais avaliaram a preocupação com o ganho de peso na gestação. A atitude materna em relação ao ganho de peso é fortemente influenciada pelo tamanho corporal pré-gestacional: mulheres magras tendem a ter atitudes positivas, e mulheres obesas atitudes negativas ${ }^{7}$. Atitudes negativas em relação ao ganho de peso foram encontradas até mesmo entre mulheres que ganharam peso dentro da faixa recomendada ${ }^{8}$. Esse estudo ${ }^{8}$, entre outros ${ }^{9,10}$, utilizou a Escala de Atitudes em relação ao Ganho de Peso na Gestação (AGPG) ${ }^{11}$ para avaliação das questões corporais.

No Brasil, os trabalhos sobre gestação e ganho de peso focam em aspectos como: retenção de peso no período pós-parto ${ }^{12}$, peso do recém-nascido ${ }^{13}$, depressão na gestação e peso ao nascer ${ }^{14}$, impacto da orientação dietética e ganho de peso ${ }^{15}$, e estado nutricional materno ${ }^{16}$. Apenas dois trabalhos avaliaram a preocupação com o ganho de peso na gestação.

Soares et al. ${ }^{17}$ avaliaram a preocupação com a forma e o peso corporais na gestação de adultas no Rio Grande do Sul utilizando o Eating Disorder Examination Questionnaire (EDE-Q) e constataram uma redução da preocupação com a forma e o peso durante a gestação. Uma explicação, segundo os autores, é que as mulheres que apresentavam maior preocupação antes da gravidez podem tornar-se temporariamente menos preocupadas com calorias, peso e forma em função do foco no desenvolvimento do feto e na saúde. Menezes e Domingues ${ }^{18}$ traduziram e aplicaram a AGPG em 132 adolescentes gestantes na cidade de Goiânia - GO, e constataram que a maioria teve atitudes positivas em relação ao ganho de peso.

A AGPG é o único instrumento que avalia atitudes em relação ao ganho de peso especificamente em gestantes. A escala foi desenvolvida por Palmer et al..$^{11}$ e conta com 18 afirmações que expressam atitudes de gestantes em relação ao seu ganho de peso.

Considerando-se que avaliar as atitudes em relação ao ganho de peso tem importância para a evolução de uma gestação saudável, o uso de instrumentos válidos se faz necessário. Como o estudo nacional que usou previamente a AGPG incluiu apenas uma tradução, o objetivo deste trabalho é apresentar o processo de adaptação transcultural da AGPG com o uso das equivalências semântica, de item, operacional e de mensuração.

\section{Métodos}

Trata-se de um estudo transversal, metodológico, cujo processo de adaptação transcultural seguiu as etapas recomendadas ${ }^{19}$ e descritas detalhadamente por alguns pesquisadores ${ }^{20}$ e aplicadas por diversos autores em escalas psicológicas ${ }^{21,22}$.

Para a realização da adaptação transcultural da AGPG, obteve-se a autorização de seus autores e, na sequência, foi realizada a avaliação da equivalência semântica, que envolveu tradução, retrotradução e avaliação da compreensão da escala por especialistas da área de Nutrição. A tradução do instrumento original do idioma inglês para o português foi realizada por um nutricionista fluente em inglês e português, e a versão traduzida por Menezes e Domingues ${ }^{18}$ foi considerada uma das versões, totalizando a obtenção de duas versões, conforme preconizado ${ }^{20}$. 
Essas duas versões foram discutidas entre duas nutricionistas com experiência em imagem corporal, comportamentos alimentares e adaptação de instrumentos para se concluir uma versão que apresentasse melhor equivalência conceitual e linguagem mais adequada ao público-alvo. A partir da verificação da manutenção do significado e das ideias originais, obteve-se então a versão 1 para iniciar a etapa de avaliação de compreensão.

A avaliação da equivalência semântica da versão 1 foi realizada por uma equipe de 14 nutricionistas ( 5 deles com experiência em imagem corporal e comportamentos alimentares), que, recebendo o questionário por e-mail, deveriam enviá-lo de volta pelo mesmo meio, respondendo o quanto foi compreendido de cada questão, utilizando a classificação: 1 - não entendi nada; 2 - entendi só um pouco; 3 - entendi mais ou menos; 4 - entendi quase tudo, mas tive algumas dúvidas; ou 5 - entendi perfeitamente e não tenho dúvidas. Também foi solicitado a eles que, caso não tivessem compreendido alguma questão e/ou a linguagem não parecesse adequada, que fizessem comentários explicando os motivos.

Para avaliação da equivalência de item — que consiste na exploração do construto de interesse, do peso, da relevância e da pertinência atribuídos a seus domínios no país de origem e no contexto sociocultural em que será aplicado - foi realizada discussão entre especialistas da área de Nutrição a fim de verificar se a definição dos conceitos de interesse seria relevante e pertinente ao novo contexto ao qual estava sendo adaptado - etapa que também envolveu discussão com nutricionistas especialistas em imagem corporal e comportamento alimentar. Catorze nutricionistas (apenas um do gênero masculino) foram convidados a participar da avaliação da versão 1 : cinco deles trabalham com transtornos alimentares, cinco trabalham na área clínica hospitalar e quatro são da área acadêmica. Um médico ginecologista e obstetra, supervisor do atendimento a gestantes adolescentes em um hospital público na cidade de São Paulo, também participou da avaliação conceitual e de itens. A partir dessa avaliação e dos comentários e sugestões dos especialistas, foi gerada a versão final da AGPG.

A equivalência operacional envolve a avaliação da compreensão das questões, tempo de preenchimento e formato da escala. Da mesma maneira adotada em uso prévio no Brasil ${ }^{18}$, optou-se por excluir a opção de resposta "nem discordo, nem concordo", com o objetivo de obter uma posição mais clara de discordância ou concordância da participante em relação a cada afirmação. Essa equivalência foi verificada por meio de um pré-teste da escala já adaptada em gestantes adultas de baixo risco $(n=15)$ atendidas na Liga Acadêmica de Assistência Pré-natal da Clínica Obstétrica do Hospital das Clínicas da Faculdade de Medicina da Universidade de São Paulo.
Essas gestantes foram convidadas a preencher a escala durante a espera pelo atendimento. O tempo médio de preenchimento foi de cinco minutos, com compreensão adequada da escala.

Com relação à equivalência de mensuração, esta envolve comparação entre as propriedades psicométricas do instrumento original e de sua nova versão. No presente estudo, essa avaliação envolveu a consistência interna do instrumento e uma análise fatorial exploratória. Para as medidas dessa equivalência, a AGPG foi aplicada em gestantes adultas de baixo risco $(n=180)$ também atendidas na Liga Acadêmica de Assistência Pré-natal da Clínica Obstétrica do Hospital das Clínicas da Faculdade de Medicina da Universidade de São Paulo e convidadas a preencher a escala durante a espera pelo atendimento. Foram consideradas elegíveis para o estudo gestantes matriculadas neste serviço e que se encontrassem entre a $12^{\mathrm{a}}$ e $38^{\mathrm{a}}$ semanas de gestação, e inelegíveis as analfabetas, portadoras de algum transtorno psiquiátrico ou usuárias de drogas.

\section{Escala de Atitudes em Relação}

ao Ganho de Peso durante a Gestação

A AGPG foi desenvolvida nos Estados Unidos e testada com 29 gestantes para determinar se as atitudes em relação à magreza afetariam o ganho de peso durante a gravidez. A escala consistia de 40 afirmações sobre diferentes atitudes de gestantes em relação ao seu ganho de peso. A validade do conteúdo ou adequação das afirmações foi julgada por 30 avaliadores, e as 20 questões consideradas mais apropriadas foram compiladas em uma escala do tipo Likert. Após a realização de um pré-teste com gestantes no último trimestre de gestação, chegou-se à versão final de 18 afirmações ${ }^{11}$.

A AGPG é, portanto, composta por 18 afirmações que expressam atitudes de gestantes em relação ao seu ganho de peso. É uma escala de autopreenchimento, respondida na forma de escala Likert de pontos $(1$ - concordo fortemente a 4 - discordo fortemente). A pontuação total varia de 18 -atitude muito negativa a 72 -atitude muito positiva em relação ao ganho de peso na gestação.

\section{Análises estatísticas}

As análises dos dados foram conduzidas com o software SPSS 17.0 - Statistical Package for Social Sciences (SPSS, Chicago, Il, USA). Para todas elas foi adotado nível de significância de $5 \%$. As respostas dos experts foram avaliadas por frequência, e os dados de idade, idade gestacional e escores das gestantes na AGPG são apresentados como tendência central e desvio padrão. Para a equivalência de mensuração, a consistência interna foi avaliada pelo Alpha de Cronbach e Split-Half pela fórmula de Spearman-Brown. 
Uma análise fatorial exploratória foi realizada por meio de uma análise de componentes principais, seguida de rotação Varimax. Soluções foram testadas para atender aos critérios: Kaiser-Meyer-Olkin $\geq 0,5$; teste de esfericidade de Bartlett $\leq 0,05$; comunalidade de cada variável $\geq 0,35$; eigenvalues para todos os fatores $\geq 1$. Cada fator deveria ainda explicar pelo menos $5 \%$ da variância total, e cada variância deste fator deveria ter carga fatorial $\geq 0,4^{23}$. O scree plot foi examinado para o ponto de transição na função para se definir o número adequado de fatores na escala.

\section{Considerações éticas}

A adaptação transcultural da AGPG se insere como parte preliminar do projeto intitulado "Duas grandes transformações ao mesmo tempo: atitudes em relação à alimentação e ao corpo em gestantes adolescentes", que foi aprovado pelo Comitê de Ética e Pesquisa (CEP) da Faculdade de Saúde Pública da Universidade de São Paulo e pela Comissão de Ética para Análise de Projetos de Pesquisa (CAPPesq) do Hospital das Clínicas da Faculdade de Medicina da Universidade de São Paulo - Protocolo n ${ }^{\circ}$ 353/12 em 4 de fevereiro de 2013; as gestantes participantes assinaram o Termo de Consentimento Livre e Esclarecido.

\section{Resultados}

A versão 1 e a versão final da AGPG podem ser visualizadas no link https://docs.google.com/ file/d/0Bw3P3XNCt8qZX0RFT0pMMTdTQ0k/edit?pli=1.

Com relação à versão 1 avaliada pelos especialistas, cinco questões tiveram $100 \%$ de compreensão perfeita e, portanto, foram mantidas como em sua primeira versão adaptada. As demais questões tiveram graus variados de compreensão ou geraram algumas dúvidas, conforme a Tabela 1 .

As 18 questões com graus variados de compreensão foram discutidas com os experts em relação às dúvidas e sugestões para expressões e palavras de melhor entendimento e que traduzissem de maneira fidedigna e com clareza a ideia proposta no instrumento original.

As afirmações que mencionavam faixas de ganho de peso (2, 4 e 10) foram convertidas do original pounds para quilogramas e suscitaram dúvidas do que seria normal ou excessivo de acordo com as recomendações para a população brasileira. A faixa de ganho de peso para mulheres eutróficas foi alterada para o que representa o ganho recomendado em âmbito clínico para tais mulheres $^{24} \mathrm{e}$ a faixa referente a ganhos considerados excessivos foi mantida segundo o Institute of Medicine ${ }^{25}$, referência adotada no ambulatório onde foram coletados os dados.

As modificações sugeridas pelos experts foram feitas, conforme cada caso, e discutidas, resultando na versão final do instrumento. A evolução do processo de adaptação transcultural com relação às versões do instrumento pode ser visualizada no quadro disponível no link acima.

A versão resultante da avaliação dos profissionais foi retraduzida para o inglês por um tradutor bilíngue, de forma independente, conforme a recomendação ${ }^{19}$. A tradução e a retrotradução foram discutidas pelos autores deste trabalho e discrepâncias não foram encontradas.

As participantes da etapa de equivalência de mensuração foram 180 gestantes - número definido com o objetivo de se obter pelo menos 10 respondentes para cada uma das questões da escala ${ }^{26}$. O tempo médio de preenchimento da escala foi de 5 minutos, avaliado no pré-teste, correspondente à equivalência operacional.

Essas gestantes tinham média de 29,6 anos ( $\mathrm{DP}=5,2)$ e idade gestacional média de 25,7 semanas $(D P=7,1)$. A pontuação média obtida por esse grupo na AGPG foi de 54,5 (DP=6,6). A mediana foi de 54. Setenta e sete gestantes pontuaram abaixo da mediana e $103(57,2 \%)$ acima dela; ainda, $27,8 \%(\mathrm{n}=50)$ estiveram no quartil de pontuação mais baixa e $22,2 \%(n=40)$ no quartil mais alto de pontuação. O Alpha de Cronbach da AGPG foi de 0,7 e o Split-Half também 0,7 .

A análise fatorial exploratória obteve alta correlação no teste de esfericidade de Barlett $\left[\left(\chi^{2}(153)=699, \mathrm{p}<0,001\right]\right.$, e a medida de adequação de Kaiser-Meyer-Olkin de 0,7; o scree plot apontou quatro fatores para a escala. A solução de três ou cinco fatores foi também testada, mas com cinco fatores, dois deles teriam apenas duas questões, e a solução de três fatores teve menores comunalidades e menor variância explicada.

A análise definida com extração de quatro fatores encontrou variância total explicada de 51,4\%; atendeu aos critérios propostos e encontrou ainda uma matriz de componentes que fez sentido conceitual (Tabela 2).

Dessa forma, os fatores foram nomeados de acordo com as afirmativas em cada um deles. O fator 1 , nomeado "incômodo com o ganho de peso", inclui 8 questões $(3,4,9,12,13,14,16$ e 17), que contam para $17,2 \%$ da variância (eigenvalue 3,6); o fator 2, nomeado "preocupação com o peso", inclui 5 questões $(1,2,5,8,15)$, que contam para $11,5 \%$ da variância (eigenvalue 2,2); o fator 3, nomeado "bem-estar com a gravidez", inclui 3 questões $(6,7$ e 11$)$, que contam para 11,4\% da variância (eigenvalue 2.0); por fim, o fator 4, nomeado "ganharia mais peso se necessário", inclui 2 questões (10 e 18), que contam para 11,4\% da variância (eigenvalue 1,5 ).

A consistência interna para os fatores foi calculada e encontrou Alpha de Cronbach 0,8 para "incômodo com o ganho de peso", 0,4 para "preocupação com o ganho de peso", 0,7 para "bem-estar com a gravidez" e 0,8 para "ganharia mais peso se necessário". 
Tabela 1. Frequência de respostas dos experts $(n=15)$ segundo opções de compreensão das afirmações da Escala de Atitudes em Relação ao Ganho de Peso na Gestação

\begin{tabular}{|c|c|c|c|c|c|}
\hline 1. Eu me preocupo se posso ficar gorda durante esta gravidez. & & & & 33,3 & 66,7 \\
\hline 3. Eu estou tentando manter meu peso baixo para não parecer grávida. & & & & 13,3 & 86,7 \\
\hline 4. Eu gostaria de ganhar entre 5 e $9 \mathrm{~kg}$ durante esta gravidez. & & & & & 100,0 \\
\hline 6. Eu acho que uma mulher grávida é bonita. & & & & 13,3 & 86,7 \\
\hline 7. Eu me orgulho de parecer grávida. & & & & 20,0 & 80,0 \\
\hline 8. Eu gosto de ser capaz de ganhar peso para esta gravidez. & 6,7 & 6,7 & 6,7 & 26,6 & 53,3 \\
\hline 9. Eu estov envergonhada do quanto tenho engordado durante esta gravidez. & & & 6,7 & 6,7 & 86,7 \\
\hline 12. 0 peso que eu ganho durante a gravidez me faz sentir não atraente. & & & 6,7 & 20,0 & 73,3 \\
\hline 13. Eu fico envergonhada toda vez que um profissional de saúde me pesa. & & & & & 100,0 \\
\hline 14. Eu me incomodo por não poder usar a roupa da moda enquanto eu estou grávida. & & & & 6,7 & 93,3 \\
\hline $\begin{array}{l}\text { 15. Eu acho que a mulher deve ser especialmente cuidadosa quanto a ficar gorda } \\
\text { durante a gravidez. }\end{array}$ & & 6,7 & 6,7 & 26,7 & 60,0 \\
\hline 16. Se eu ganho muito peso em um mês, tento não engordar no mês seguinte. & & & & & 100,0 \\
\hline 17. Logo antes de ir ao médico, tento não comer nada. & & & & 6,7 & 93,3 \\
\hline 18. Eu ganharia 16 kg se isto significasse que meu bebê seria mais saudável. & & 6,7 & & 6,7 & 86,7 \\
\hline Total & 0,4 & 1,5 & 1,5 & 11,5 & 85,2 \\
\hline
\end{tabular}

1 - Não entendi nada; 2 - Entendi só um pouco; 3 - Entendi mais ou menos; 4 - Entendi quase tudo, mas tive algumas dúvidas; 5 - Entendi perfeitamente e não tenho dúvidas.

Tabela 2. Resultados da análise fatorial exploratória para a Escala de Atitudes em Relação ao Ganho de Peso na Gestação

\begin{tabular}{|c|c|c|c|c|}
\hline Item & Fator 1 & Fator 2 & Fator 3 & Fator 4 \\
\hline 1 & 0,21 & 0,67 & $-0,14$ & $-0,03$ \\
\hline 2 & 0,46 & $-0,51$ & $-0,01$ & 0,26 \\
\hline 3 & 0,55 & 0,01 & 0,15 & 0,01 \\
\hline 4 & 0,57 & 0,01 & $-0,11$ & 0,35 \\
\hline 5 & 0,03 & 0,46 & 0,13 & 0,44 \\
\hline 6 & 0,21 & $-0,17$ & 0,78 & 0,05 \\
\hline 7 & 0,17 & $-0,17$ & 0,76 & 0,10 \\
\hline 8 & 0,07 & 0,43 & 0,084 & 0,40 \\
\hline 9 & 0,60 & $-0,03$ & 0,088 & 00,8 \\
\hline 10 & 0,03 & 0,04 & 0,204 & 0,81 \\
\hline 11 & 0,02 & 0,22 & 0,753 & 0,11 \\
\hline 12 & 0,56 & 0,30 & 0,136 & $-0,23$ \\
\hline 13 & 0,78 & 0,22 & 0,213 & $-0,05$ \\
\hline 14 & 0,44 & 0,30 & 0,210 & $-0,25$ \\
\hline 15 & 0,12 & 0,73 & $-0,077$ & 0,06 \\
\hline 16 & 0,52 & 0,32 & $-0,246$ & 0,14 \\
\hline 17 & 0,62 & 0,02 & 0,066 & $-0,09$ \\
\hline 18 & $-0,07$ & $-0,05$ & $-0,007$ & 0,81 \\
\hline
\end{tabular}

Fator 1: incômodo com $\circ$ ganho de peso; fator 2: preocupação com o peso; fator 3: bem-estar com a gravidez; fator 4: ganharia mais peso se necessário.

\section{Discussão}

O processo de adaptação transcultural da AGPG foi realizado segundo as recomendações ${ }^{19,20}$, encontrando boa equivalência em todas as etapas. As alterações de termos, verbos e organização de frases realizadas na avaliação das equivalências semântica e de itens foram necessárias para garantir uma linguagem de mais fácil entendimento, preservando a compreensão do conteúdo expresso na versão original - medida que está de acordo com a proposição de que se deve avaliar criticamente o instrumento para capturar a intenção do instrumento original, mesmo modificando palavras ${ }^{21,22}$. Houve boa compreensão e facilidade por parte das gestantes ao responder o instrumento, atestando a equivalência operacional, o que mostra que pode inclusive ser utilizado em gestantes de baixa escolaridade.

Considerando que quanto menor a pontuação mais negativa a atitude em relação ao ganho de peso durante a gestação, para os escores encontrados nesta aplicação, perto de $30 \%$ das participantes tiveram atitudes mais negativas e aproximadamente $20 \%$ atitudes mais positivas. 
A consistência interna da escala como um todo teve Alpha de Cronbach geral adequado e Split-Half igual ao encontrado no estudo original $(0,7)^{11}$ e ao avaliado no trabalho brasileiro $(0,7)^{18}$.

Como mencionado anteriormente, o uso anterior no Brasil apenas traduziu a escala sem adaptação transcultural e realizou apenas medida de consistência interna. No entanto, somente um processo de adaptação transcultural de qualidade é capaz de tornar um instrumento apto a ser utilizado em outro contexto cultural $^{27,19}$; e é importante a descrição detalhada das etapas de operacionalização na adaptação de um instrumento ${ }^{22}$. O presente estudo cumpre essa recomendação, adaptando a escala a partir da avaliação de uma série de equivalências e realizando ainda uma análise fatorial exploratória, não realizada no estudo original ou no brasileiro ${ }^{11,18}$. Esta avaliação encontrou que a solução de quatro fatores mostra bom resultado psicométrico. Apenas a consistência interna do fator 2 foi baixa. Tal achado não permite nenhuma comparação, uma vez que a escala não foi proposta em fatores por seus autores nem avaliada dessa maneira previamente. Assim, o uso com os quatro fatores aqui sugeridos merece cautela.

Outras equivalências de mensuração não foram realizadas nesta primeira avaliação da AGPG. Uma confiabilidade "teste-reteste" não teria validade, pois seria esperado que as respostas fossem diferentes em momentos diferentes de aplicação, uma vez que a população em questão está em um processo de transformação que é a gestação. Análises futuras podem objetivar a avaliação das demais equivalências (convergente, discriminante, concorrente), a fim de verificar sua validade externa e reprodutibilidade. De qualquer forma, como a AGPG é a única escala específica localizada para a avaliação de um aspecto específico da imagem corporal de gestantes (preocupação com ganho de peso), sua adaptação e a avaliação de propriedades psicométricas preliminares, aqui apresentadas, atestam sua validade e utilidade.

Instrumentos específicos de avaliação da imagem corporal, em seus componentes perceptivo e atitudinal, construídos para gestantes não são disponíveis. Estudos que de alguma forma avaliaram o construto nessa população usam uma variedade de instrumentos, incluindo alguns construídos pelo estudo ${ }^{28}$ e escalas validadas que foram desenvolvidas para o público em geral ${ }^{29}$, eventualmente com algumas adaptações para o público gestante ${ }^{30-33}$. Uma vez que esse momento da vida da mulher pode implicar em alterações da imagem corporal — muitas vezes de maneira negativa - especialmente na cultura atual que cobra "esbelteza" até mesmo durante uma gestação, ter um instrumento validado para o cenário brasileiro é um avanço importante.

Conforme proposta das quatro subescalas aqui apresentadas, aspectos positivos, como o bem-estar com a gravidez e a possibilidade de ganhar mais peso inclusive, se isso for para o bem do bebê e da gestação, podem ser avaliados pela AGPG. Acredita-se que tais fatores possam se relacionar com o fato de seguir as recomendações para uma gestação saudável, incluindo exames pré-natais, cuidados específicos com a alimentação, medicação, etc., pois é sabido, por exemplo, que a ingestão inadequada de nutrientes é frequente em mulheres em idade fértil e em gestantes, porque elas não alteram sua ingestão alimentar durante a gravidez, agravando essas deficiências ${ }^{34}$.

Por outro lado, as subescalas 1 e 2 permitem a avaliação de aspectos negativos, como a preocupação com o peso (que pode ser exagerada) e o incômodo com o ganho de peso. Tais atitudes podem se relacionar com o não seguimento das recomendações, como uso de suplementos específicos, cuidados com a alimentação e prática de atividade física. Estes dois últimos podem se apresentar de forma restritiva e exagerada mesmo se contraindicados em função do foco da mãe ser mais sua aparência do que a saúde de seu bebê e o bom desenvolvimento da gestação.

Em contrapartida, como a proporção de gestantes com excesso de ganho de peso é alta ${ }^{35}$, a escala AGPG pode ainda ser útil para rastrear gestantes em risco de ganho ponderal excessivo, por ausência de preocupação com o peso, e que podem inclusive ter iniciado a gestação com sobrepeso. Tal observação é importante uma vez que o início da gestação com excesso de peso e o ganho de peso gestacional estão associados com maior retenção de peso 12 meses após o parto ${ }^{36}$.

Mulheres que já são preocupadas com o peso e com a forma corporal podem ter na gravidez um aumento dessa ansiedade relacionada ao peso ${ }^{37}$. Na cultura atual — em razão da associação da magreza com beleza, sucesso e inteligência - muitas mulheres adotam hábitos alimentares inadequados e algumas desenvolvem comportamentos transtornados e transtornos alimentares ${ }^{38}$. Como gestantes são majoritariamente mulheres jovens, essa fase pode representar risco de surgimento de insatisfação corporal e dos comportamentos alimentares mencionados ${ }^{38,37}$. Transtornos alimentares na gestação são razoavelmente comuns, com prevalência estimada de 0,1 a $4,8 \%{ }^{39}$, sendo a gestação um período de alto risco para o início do transtorno de compulsão alimentar ${ }^{39}$. Sintomas de transtornos alimentares, como compulsão e vômitos autoinduzidos, podem diminuir durante a gestação, comparados ao período pré-gestacional ${ }^{40}$; no entanto, estudos mostram que esses sintomas melhoram durante a gestação, mas pioram no período pós-parto ${ }^{41,42}$. Preocupações com o peso e a forma física são altamente prevalentes também no período pós-parto e podem representar risco para o desenvolvimento de comportamentos alimentares inadequados ${ }^{40}$.

Como perspectiva futura, uma análise da equivalência convergente da AGPG com outras escalas que avaliam 
satisfação corporal, como a Body Shape Questionnaire $(\mathrm{BSQ})^{33}$ e a Body Attitude Questionnaire (BAQ) ${ }^{43}$, as quais apresentam versões validadas para o português, pode ser útil. Pode ser de ajuda também uma tentativa de validade concorrente com o estado nutricional, imaginando-se que aquelas mais pesadas para a idade gestacional podem ser mais insatisfeitas. Essas análises estarão em andamento a partir da validade atestada para a AGPG.

Conclui-se que a AGPG foi adaptada de forma satisfatória e tem propriedades psicométricas adequadas para uso com gestantes que falam português. Uma vez que escalas específicas para avaliação da imagem corporal em gestantes, de forma ampla, não existem, a disponibilidade dessa escala adaptada transculturalmente se apresenta como possibilidade que permite não apenas a avaliação de atitudes para com o ganho de peso mas também análises de associação com parâmetros clínicos, nutricionais, de evolução da gestação e de risco para transtornos alimentares. Tal instrumento pode ser útil, portanto, em avaliações de acompanhamento pré-natal, a fim de auxiliar a detecção e a prevenção de comportamentos disfuncionais durante a gestação e melhor direcionar a assistência à saúde da mãe e do concepto durante essa fase.

\section{Referências}

1. Araújo NM, Salim NR, Gualda DM, Silva LC. Corpo e sexualidade na gravidez. Rev Esc Enferm USP. 2012;46(3):552-8.

2. Riechelmann JC. A mulher atual: perspectivas frente à gestação. In: Zugaib M, Tedesco JJ, Quayle J, editores. Obstetrícia psicossomática. São Paulo: Atheneu; 1997. p. 40-53.

3. Skouteris H, Carr R, Wertheim EH, Paxton SJ, Duncombe D. A prospective study of factors that lead to body dissatisfaction during pregnancy. Body Image. 2005;2(4):347-61.

4. Heinberg L, Guarda AS. Body image issues in obstetrics and gynecology. In: Cash TF, Pruzinsky T, editors. Body images: development, deviance, and change. New York: Guilford; 2002. p. 3-20.

5. Sumner A, Waller G, Killick S, Elstein M. Body image distortion in pregnancy: a pilot study of the effects of media images. J Reprod Infant Psychol. 1993; 11 (4):203-8.

6. Gow RW, Lydecker JA, Lamanna JD, Mazzeo SE. Representations of celebrities' weight and shape during pregnancy and postpartum: a content analysis of three entertainment magazine websites. Body Image. 2012;9(1):172-5.

7. Copper RL, DuBard MB, Goldenberg RL, Oweis Al. The relashionship of maternal attitude toward weight gain to weight gain during pregnancy and low birth weight. Obstet Gynecol. 1995;85(4):590-5.

8. DiPietro JA, Millet S, Costigan KA, Gurewitsch E, Caulfield LE. Psychosocial influences on weight gain attitudes and behaviors during pregnancy. J Am Diet Assoc. 2003;103(10):1314-9.

9. Stevens-Simon C, Nakashima I, Andrews D. Weight gain attitudes among pregnant adolescents. J Adolesc Health. 1993;14(5):369-72.

10. Gutierrez YM. Cultural factors affecting diet and pregnancy outcome of Mexican American adolescents. J Adolesc Health. 1999;25(3):227-37.

11. Palmer JL, Jennings GE, Massey L. Development of an assessment form: attitude toward weigh gain during pregnancy. J Am Diet Assoc. 1985;85(8):946-9.

12. Martins AP, Benício MH. Influência do consumo alimentar na gestação sobre a retenção de peso pós-parto. Rev Saúde Pública. $2011 ; 45(5): 870-7$.

13. Surita FG, Suarez MB, Siani S, Silva JL. [Factors associated with low birth weight among adolescents in the Brazil Southeast region]. Rev Bras Ginecol Obstet. 201 1;33(10):286-91. Portuguese.

14. Thiengo DL, Pereira PK, Santos JF, Cavalcanti MT, Lovisi GM. Depressão durante a gestação e os desfechos na saúde do recémnascido: coorte de mães atendidas em unidade básica de saúde. J Bras Psiquiatr. 2012;61 (4):214-20.
15. Vitolo MR, Bueno MS, Gama CM. [Impact of a dietary counseling program on the gain weight speed pf pregnant women attended in a primary care service]. Rev Bras Ginecol Obstet. $2011 ; 33(1): 13$ 9. Portuguese.

16. Melo MI, Souza Al, Figueiroa JN, Cabral-Filho JE, Benício MH, Batista-Filho M. Estado nutricional de gestantes avaliado por três diferentes métodos de classificação antropométrica. Rev Nutr. $2011 ; 24(4): 585-92$.

17. Soares RM, Nunes MA, Schmidt MI, Giacomello A, Manzolli P, Camey $S$, et al. Inappropriate eating behaviors during pregnancy: prevalence and associated factors among pregnant women attending primary care in southern Brazil. Int J Eat Disord. 2009;42(5):387-93.

18. Menezes IH, Domingues MH. Principais mudanças corporais percebidas por gestantes adolescentes assistidas em serviços públicos de saúde de Goiânia. Rev Nutr. 2004;17(2):185-94.

19. Herdman M, Fox-Rushby J, Badia X. A model of equivalence in the cultural adaptation of $H R Q \circ L$ instruments: the universalist approach. Qual Life Res. 1998;7(4):323-35.

20. Reichenheim ME, Moraes CL. Operacionalização de adaptação transcultural de instrumentos de aferição usados em epidemiologia. Rev Saúde Pública. 2007;41 (4):665-73.

21. Barillari ML, Teixeira PC, Hearst N, Kotait MS, Cordás TA, Conti MA. Adaptação transcultural preliminar do Children's Eating Attitude Test (Cheat) para o idioma português. Rev Bras Saúde Matern Infant. $2011 ; 11(4): 437-44$.

22. Conti MA, Slater $B$, Latorre MR. Validação e reprodutibilidade da Escala de Evaluación de Insatisfación Corporal para Adolescentes. Rev Saúde Pública. 2009;43(3):515-24.

23. Hair JF. Multivariate data analysis with readings. Englewood Cliffs: Prentice Hall; 1995.

24. Vitolo MR. Nutrição: da gestação ao envelhecimento. Rio de Janeiro: Rubio; 2008.

25. National Academies. Institute of Medicine. National Research Council [Internet]. Weight gain during pregnancy: rexamining the guidelines. Washington, DC: The National Academies Press; 2009 [cited 2012 Jul 11]. Available from: <http://www.iom. edu/ /media/Files/Report\%20Files/2009/Weight-Gain-DuringPregnancy-Reexamining-the-Guidelines/Report\%20Brief\%20-\%20 Weight\%20Gain\%20During\%20Pregnancy.pdfhttp://www.iom. edu/Reports/2009/Weight-Gain-During-Pregnancy-Reexaminingthe-Guidelines.aspx/> 
26. Nunnaly JC, Bernstein IH. Psychometric theory. New York: McGrawHill; 1994.

27. Guillemin F, Bombardier C, Beaton D. Cross-cultural adaptation of health-related quality of life measures: literature review and proposed guidelines. J Clin Epidemiol. 1993;46(12):1417-32.

28. Davies K, Wardle J. Body image and dieting in pregnancy. J Psychosom Res. 1994;38(8):787-99.

29. Metha UJ, Siega-Riz AM, Herring AH. Effect of body image on pregnancy weight gain. Matern Child Health J. $2011 ; 15(3): 324-32$.

30. Duncombe D, Wertheim EH, Skouteris H, Paxton SJ, Kelly L. How well do women adapt to changes in their body size and shape across the course of pregnancy? J Health Psychol. 2008;13(4):503-15.

31. Clark A, Skouteris H, Wertheim EH, Paxton SJ, Milgrom J. The relationship between depression and body dissatisfaction across pregnancy and the postpartum: a prospective study. J Health Psychol. 2009; 14(1):27-35.

32. Fox $P$, Yamaguchi $C$. Body image change in pregnancy: a comparison of normal weigh na overweigh primigravidas. Birth. 1997;24(1):35-40.

33. Cooper PJ, Taylor M, Cooper Z, Fairburn CG. The development and validation of the Body Shape Questionnaire. Int J Eat Disord. 1987;6(4):485-94.

34. dos Santos Q, Sichieri R, Marchioni DM, Verly Junior E. Brazilian pregnant and lactating women do not change their food intake to meet nutritional goals. BMC Pregnancy Childbirth. 2014;14(1):186.

35. Carvalhaes MA, Gomes CB, Malta MB, Papini SJ, Parada CM. [Prepregnancy overweight is associated with excessive weight gain during pregnancy]. Rev Bras Ginecol Obstet. 2013;35(1 1):523-9. Portuguese.
36. Nast M, Oliveira A, Rauber F, Vitolo MR. [Excessive gestational weight gain is risk factor for overweight among women]. Rev Bras Ginecol Obstet. 2013;35(12):536-40. Portuguese.

37. James DC. Eating disorders, fertility, and pregnancy: relationships and complications. J Perinat Neonatal Nurs. $2001 ; 15(2): 36-48$.

38. Broussard B. Psychological and behavioral traits associated with eating disorders and pregnancy: a pilot study. J Midwifery Womens Health. 2012;57(1):61-6.

39. Bulik CM, Von Holle A, Hamer R, Knoph Berg C, Torgersen L, Magnus $P$, et al. Patterns of remission, continuation and incidence of broadly defined eating disorders during early pregnancy in the Norwegian Mother and Child Cohort Study (MoBa). Psychol Med. 2007;37(8): 1 109-18.

40. Nunes MA, Pinheiro AP, Hoffmann JF, Schmidt MI. Eating disorders symptoms in pregnancy and postpartum: a prospective study in a disadvantaged population in Brazil. Int J Eat Disord. 2014;47(4):426-30.

41. Blais MA, Becker AE, Burwell RA, Flores AT, Nussbaum KM, Greenwood DN, et al. Pregnancy: outcome and impact on symptomatology in a cohort of eating-disordered women. Int J Eat Disord. 2000;27(2): 140-9.

42. Rocco PL, Orbitello B, Perini L, Pera V, Ciano RP, Balestrieri M. Effects of pregnancy on eating attitudes and disorders: a prospective study. J Psychosom Res. 2005;59(3):175-9.

43. Scagliusi FB, Polacow VO, Cordás TA, Coelho D, Alvarenga M, Philippi ST, et al. Psychometric testing and applications of the Body Attitudes Questionnaire translated into Portuguese. Percept Mot Skills. 2005;101(1):25-41. 